\title{
A DEFICIÊNCIA INTELECTUAL: HISTÓRIA E ESTIGMATIZAÇÃO IMPOSTA AS PESSOAS AO LONGO DOS TEMPOS
}

\author{
INTELLECTUAL DISABILITY: HISTORY AND STIGMATIZATION IMPOSED ON \\ PEOPLE OVER TIME
}

\section{Madson Márcio de Farias Leite ${ }^{2}$}

RESUMO: A história das pessoas com deficiência passou por algumas mudanças de estigmas ao longo dos tempos, os motivos foram os mais diferentes possíveis isso em virtude da falta de conhecimento da causa e o preconceito ao diferente, dessa forma perdurou por várias décadas essa condição. Os sujeitos ao qual eram acometidos pela deficiência intelectual passaram por um processo de várias mudanças decorrentes da falta de conhecimento de quais limitações eram acarretadas a vida daqueles que eram diagnosticados como tendo deficiência intelectual. O presente estudo vem apresentar a história e estigmatização imposta as pessoas com deficiência intelectual ao longo dos tempos, descrevendo como objetivo de investigação a análise da estigmatização que as pessoas com deficiência intelectual passaram durante longos períodos da história. E assim buscando possibilidades de causa para arrefecimento a todos aqueles que fossem acometidos por essa deficiência, possibilitando informações e conhecimentos na proporção da extinção de qualquer tipo de ojeriza a vida daqueles com deficiência. A metodologia utilizada foi a pesquisa bibliográfica que esquadrinhou uma base teórica na história do percurso dessa causa através de descritos sobre todo o processo desde o nascimento ao desenvolvimento desses sujeitos ao longo de sua existência. Portanto ao descrever sobre preconceitos vividos por aqueles com deficiência é possível apresentar alguns avanços dessa luta durante os dias atuais no que se refere a melhor aceitação e uma qualidade de vida condizente a todos os outros aos quais não possuem a deficiência, e dessa forma podendo ser empregados conceitos atuais e concretos de que essas pessoas possuem limitações e não impedimentos de desfrutar de tudo aquilo que venham desejar. Assim sendo o estudo conclui que através das leis, decretos e mudanças de costumes e efetivação de algumas políticas públicas essas pessoas vêm demostrando seu potencial e assim garantindo seus espaços que durante longos anos foram extintos por uma grande parcela da população simplesmente por preconceito e falta de conhecimento. De tal modo será possível que através da ciência e estudos futuros seja possível eliminar totalmente qualquer tipo de estigma ignominioso a todos aqueles que possuem deficiência intelectual em qualquer parte do mundo, dessa forma podendo possibilitar que estes possam viver de forma igualitária a qualquer sujeito sem nenhuma limitação.

Palavras-chave: Deficiência Intelectual. Estigmatização. História. Conhecimento.

\footnotetext{
${ }^{\mathrm{I}}$ Este artigo foi construído a partir da fundamentação teórica e reflete dados bibliográficos parciais da Tese em construção em Ciências da Educação pela Universidad Autónoma de Asunción - UAA, Paraguai - (Py).

2 Psicólogo e professor de Educação Física. Doutorando em Ciências da Educação pela Universidad Autónoma de Asunción - UAA, Paraguai - (Py). Mestre em Ciências da Educação pela Universidad Interamericana Paraguai - (Py). Bacharelado em Psicologia pela Universidade Federal de Alagoas-UFAL. Licenciatura em Educação Física pela Faculdade São Tomás de Aquino - FACESTA. Pós-Graduação "Lato Sensu" em nível de especialização, em Teorias e Técnicas comportamentais: Educação, Pesquisa e Terapia. Especialista em Metodologia do Ensino da Educação Física. Pós-graduação em docência do Ensino Superior Instituição: Faculdade de ensino Regional Alternativa - FERA. Pós-graduado em Educação Inclusiva Instituição: Faculdade Venda Nova do Imigrante - FAVENI. Professor da rede municipal da cidade de Pilar - AL E-mail: madsonmarcio@hotmail.com.
} 
ABSTRACT: The history of people with disabilities have undergone some changes in stigmas over time, the reasons were as different as possible, this due to the lack of knowledge of the cause and the prejudice to the different, in this way this condition lasted for several decades. The subjects who were affected by intellectual disability went through a process of several changes resulting from the lack of knowledge of what limitations were entailed in the lives of those who were diagnosed as having intellectual disability. The present study presents the history and stigmatization imposed on people with intellectual disabilities over time, describing as an objective of investigation the analysis of the stigmatization that people with intellectual disabilities went through during long periods of history. And so looking for possibilities of cause for cooling down to all those who were affected by this disability, providing information and knowledge in proportion to the extinction of any type of hates the lives of those with disabilities. The methodology used was the bibliographic research that scrutinized a theoretical basis in the history of the course of this cause through descriptions of the entire process from birth to the development of these subjects throughout their existence. Therefore, when describing prejudices experienced by those with intellectual disabilities, it is possible to describe some advances in this struggle during the present day with regard to better acceptance and a quality of life consistent with all others who do not have the disability, and thus being able to current and concrete concepts should be used that these people have limitations and not impediments to enjoy everything they come to want. Therefore, the study concludes that through laws, decrees and changes in customs and the implementation of some public policies, these people have been demonstrating their potential and thus guaranteeing their spaces that for many years were extinct by a large portion of the population simply because of prejudice and lack of knowledge. In such a way, it will be possible that through science and future studies it will be possible to totally eliminate any type of ignominious stigma to all those who have intellectual disabilities in any part of the planet, thus enabling them to live in an egalitarian way to any subject without any limitation.

Keywords: Intellectual Disability. Stigmatization. History. Knowledge.

\section{INTRODUÇÃO}

As pessoas com deficiência foram estigmatizadas ao longo dos tempos, os motivos foram os mais diversos, entre eles o não conhecimento da causa, o preconceito, o repudio do que era diferente entre outros, isso percorreu por longos períodos, muitos foram os termos e as definições utilizadas para se referir ao sujeito com Deficiência Intelectual - DI. Ao delinear sobre a DI podemos descrever que a mesma não é caracterizada como sendo uma doença, e sim uma limitação atribuída a alguns sujeitos, que em sua grande maioria apresentam o funcionamento cognitivo que não corresponde à média esperada para a idade, ocasionando dúvida aos pais em não saber qual as áreas mais afetadas diretamente no que se refere ao desempenho cerebral da criança. Dessa forma se faz necessário que essas pessoas sejam acompanhadas por profissionais de várias especializadas para que seu processo de desenvolvimento possa ser satisfatório no desempenho integral de cada indivíduo.

O objetivo da pesquisa é apresentar sobre a história e estigmatização que as pessoas com DI passaram durante longos períodos da história, buscando através da efetivação de 
algumas políticas públicas e lutas populares a concretização dos direitos negados aos mesmos durante longos períodos, dessa forma acarretado danos irreparáveis a vida de todas essas pessoas. E como metodologia utilizou a pesquisa bibliográfica que buscou uma base teórica na história do percurso dessa causa através de descritos sobre todo o processo desde o nascimento ao desenvolvimento desses sujeitos ao longo de sua existência no que se refere aos estigmas sofridos decorrentes de terem uma deficiência.

No entanto o referido estudo ocasionara contribuições no que se refere a diminuição do estigma decorrente da falta de conhecimento inerente a todos aqueles com DI, isso em virtude da sistematização de informações sobre a deficiência, podendo assim contribuir no tocante a diminuição de toda forma de redução aos avanços dessas pessoas na sua trajetória de vida.

Destacando que a informação e as pesquisas sobre essa causa vêm ganhando avanços satisfatórios no que se refere a melhores resultados no progresso desses sujeitos em todos os âmbitos aos quais eles desejem ser inseridos, tudo é possível através do conhecimento e a ciência mostrando que se existir ensinamento vai acontecer aprendizado, e assim fazendo com que qualquer forma de estgmatização que possa ocorrer seja desmistificada.

A Organização Mundial de Saúde - OMS (2010) estima que I a 3\% da população em geral tem DI. Ainda de causa desconhecida, a comunidade médica trabalha com várias linhas de pesquisa para a DI tanto em aspectos biológicos, quanto em fatores ambientais. Há que se lembrar que o transtorno tem como ponto de partida o fato de caracterizar uma alteração no desenvolvimento do cérebro do paciente e que afeta suas ramificações, sinapses e maturação. As causas podem ser originadas por fatores genéticos, problemas ocorridos durante o parto ou na gestação, prematuridade, sequelas de meningite mas, muitas vezes, em quase 50\% dos casos, não se notifica causa alguma (BRITES, 2019).

A DI é caracterizada pelo funcionamento cognitivo intelectual que não corresponde à idade esperada, ou seja, está muito abaixo do que é considerado normal. Porém não sendo impedimento para que essas crianças possam ter um desenvolvimento de forma satisfatória através do contexto educacional em contribuição com as das salas de AEE (BRITES, 2019).

A definição de DI descrita pela OMS em (2010) "antigamente era denominada como retardo mental, como sendo uma capacidade significativamente reduzida de 
compreender informações novas ou complexas e de aprender e aplicar novas habilidades (inteligência prejudicada)".

A mudança do termo passou a ser descrito como deficiência intelectual descrito em alguns documentos como o Manual diagnóstico e estatístico de transtornos mentais DSM-V tendo sua última publicação em (2014), American Association on Mental Retardation - AAMR que agora é conhecida como American Association on Intellectual and Developmental Disability - AAIDD ou seja (Associação Americana em Deficiência Intelectual e do Desenvolvimento) foi quem criou uma definição para a DI, esta foi fundada no ano de 1876 , e tendo seu primeiro presidente Séguin, este publicou vários manuais e revisões, que incidiram na seguinte ordem cronológica: 1908, 1937, 1941, 1957, 1959, 1961, 1973, 1977, 1983, 1992, 2002 e o atual manual de (2010).

A AAIDD (2010) associação de pesquisa administrada para a realização de estudos sobre a DI, tem expressivo controle nas considerações atualmente usados em diferentes partes do mundo, como também no Brasil. Dessa forma podemos ver um breve histórico cronológico das definições apresentadas pela AAIDD apud Alles, Castro e Boueri (2019, p. 52).

Em 1908 Tredgold definia como um estado de deficiência mental de nascença ou a partir de tenra idade, devido à desenvolvimento cerebral incompleto, em consequência do qual a pessoa afetada é incapaz de realizar seus deveres como membro da sociedade na posição da vida para a qual ela nasceu. Em 1937 Tredgold definia como a deficiência mental é um estado de desenvolvimento mental incompleto de um tipo e grau que o indivíduo é incapaz de se adaptar ao ambiente normal de seus companheiros, de maneira a conseguir levar sua vida independentemente de supervisão, controle ou apoio externo. Em 194I Doll definia Um estado de incompetência social obtido na maturidade, ou provável de se obter na maturidade resultante de uma parada no desenvolvimento de origem constitucional (hereditária ou adquirida); a condição é essencialmente incurável através de tratamento e irremediável através do treinamento. Em 1959 Heber O retardo mental refere-se a um funcionamento intelectual geral abaixo da média que se origina durante o período desenvolvimental e está associado a uma deficiência em uma ou mais das seguintes: áreas (I) amadurecimento, (2) aprendizagem e (3) ajustamento social. Em i96i O retardo mental refere-se a um funcionamento intelectual geral abaixo da média que se origina durante o período desenvolvimental e está associado com deficiência no comportamento adaptativo. Em 1973 Grossman definia $O$ retardo mental refere-se a um funcionamento intelectual geral significativamente abaixo da média, existente ao mesmo tempo com déficits no comportamento adaptativo e manifestado durante o período desenvolvimental. Em 1983 Grossman definia O retardo mental refere-se ao funcionamento intelectual geral significativamente abaixo da média, resultando em ou associado a deficiências concomitantes no comportamento adaptativo e manifestado durante o período de desenvolvimento. Em 1992 Luckasson et al definia $O$ Retardo mental refere-se a limitações substanciais no funcionamento atual. É caracterizado por um funcionamento intelectual significativamente abaixo da média, existente ao mesmo tempo com limitações relacionadas em duas ou mais das seguintes áreas de habilidades adaptativas aplicáveis: comunicação, autocuidado, vida doméstica, habilidades sociais, uso da comunidade, autodirecionamento, aprendizagem funcional, lazer e trabalho. Em 2002 
Luckasson et definia $O$ retardo mental é uma incapacidade caracterizada por importantes limitações, tanto no funcionamento intelectual quanto no comportamento adaptativo, está expressa nas habilidades adaptativas conceituais, sociais e práticas. Em 2010 Shogren et al definia A deficiência intelectual é caracterizada por limitações significativas tanto no funcionamento intelectual quanto no comportamento adaptativo como é expresso nas habilidades adaptativas conceituais, sociais e práticas.

O resumo descrito apresenta toda a trajetória ocorrida ao longo dos tempos sobre os descritos da pessoa com deficiência, vários debates e discursões sobre a temática levaram a mudança do termo utilizado, perpassando do nome de "deficiência mental" e "retardo mental", sendo substituído por "deficiência intelectual". Com toda essa mudança aperfeiçoou-se a visão existente a pessoa com DI, como sua forma de tratamento e percepção. $O$ conceito atual descrito na II ${ }^{\underline{a}}$ edição do manual já exibe a nomenclatura que já estava sendo empregada desde a Conferência sobre Deficiência Intelectual realizada no Canadá OMS (2004), o evento que originou a Declaração Internacional de Montreal sobre Inclusão, a alteração que encontramos na nova edição de (2010) referente a edição de (2002) é em relação a substituição do termo deficiência mental por deficiência intelectual.

De acordo a II $^{\mathbf{a}}$ edição da AAIDD a definição de deficiência intelectual é caracterizada por:

Limitações significativas tanto no funcionamento intelectual quanto no comportamento adaptativo como é expresso nas habilidades adaptativas conceituais, sociais e práticas. Essa deficiência se origina antes dos 18 anos AAIDD (2010, p. 5).

A DI proporciona percalços relacionadas ao funcionamento intelectual e adaptativos dos sujeitos, ocasionando problemas no raciocínio, habilidades sociais, aspectos relacionados ao aprendizado, na resolução de tarefas do dia a dia entre outras mais, isso sendo acarretado antes de completar 18 anos. Dessa forma se faz necessário o diagnóstico realizado de forma precoce, para que as limitações empostas pela deficiência não venha a impedir os mesmos de realizarem suas funções naturais ao longo de sua vida.

Por não se caracterizar apenas por um aspecto físico ou biológico exclusivo, a DI deve ser avaliada de forma integral no indivíduo, sendo fundamental a utilização de "testes padronizados para que sejam avaliados elementos relacionados a capacidade cognitiva ou psicométrica, como o quociente de inteligência - QI, devendo ser considerado para indivíduos com QI inferior a 70” Brasília (2018, p. 16). Os testes de QI são considerados satisfatórios na avaliação do potencial intelectual e utilizados pela Organização Mundial de Saúde (OMS), que considera quatro níveis de gravidade, de leve a profunda”, Brasília (2018, p. 17). Conforme especificado na tabela 4. 
TABELA $N^{\circ}$ r: Classificação da deficiência intelectual de acordo com o quociente de inteligência.

\begin{tabular}{|c|c|c|}
\hline CLASSIFICAÇÃO & QI & FREQUÊNCIA \\
\hline DI leve & 57 a 70 & $85 \%$ \\
\hline DI Moderada & 35 a 49 & 10\% \\
\hline DI Grave & 23 a 34 & $3 \%$ a $4 \%$ \\
\hline DI Profunda & $<20$ & I\% a $2 \%$ \\
\hline
\end{tabular}

DI: Deficiência intelectual. QI: quociente de inteligência. IM: Idade mental de um adulto. CID-ı: Classificação Internacional de Doenças para a DI de acordo com a Organização Mundial da Saúde. AAMR: American Association on Mental Retardation/Deficiency. APA: American Psychological Association (APA, 2014).

A DI passou a utilizar formas de medir através do QI as pessoas pelas quais eram acometidas pela deficiência, isso como forma de poder saber em que grau elas se encontram para assim poder interferir nos aspectos que mais sejam necessários para o processo de desenvolvimento e equiparação nas áreas mais afetadas pela deficiência. Elas passaram a ser descritas conforme as especificidades que ocorrem em cada grau da DI, segundo a AAIDD:

A DI leve é muitas vezes diagnosticada tardiamente ou nem sequer é diagnosticada, já que na maioria das vezes ela se apresenta de forma nãosindrômica ou associada a elementos dismórficos menores, que passam despercebidos durante uma avaliação médica. Ela é caracterizada por dificuldades de aprendizagem que não são muito graves. Muitos adultos com este sinal são propensos a trabalhar e adquirir boa autonomia pessoal e independência social

A DI moderada está associada com dificuldades significativas de aprendizagem. Ela se caracteriza pela aquisição de competências simples, permitindo a comunicação social, um certo grau de auto-suficiência e uma vida semiindependente. As noções básicas de leitura e escrita são raramente adquiridas. Já a DI grave é acompanhada por uma completa dependência para a realização das tarefas e ações cotidianas. Enquanto que a DI profunda é caracterizada por uma redução severa do potencial de comunicação e de mobilidade (2010, p. 12).

A DI deve ser sempre analisada de formai integral, sendo seu diagnóstico fundamental para que através dos testes possam analisar em que QI se enquadra os sujeitos, "Os testes de QI são considerados satisfatórios na avaliação do potencial intelectual e utilizados pela OMS, que considera quatro níveis de gravidade, de leve a profunda, conforme especificado na tabela $\mathrm{N}^{\circ} 4$ " Brasília (2018, p. I6).

Outro importante documento que proporcionou mudanças a vida dos sujeitos com DI foi o Manual diagnóstico e estatístico de transtornos mentais: DSM-5 (2014), publicado pela Associação Americana de Psicologia em (2014), descrevendo e definindo o que seja DI 
e quais os prejuízos causados as pessoas que forem acometidas no tocante ao desenvolvimento integral desses sujeitos.

De acordo o DSM-5, este apresenta Deficiência intelectual como sendo o (transtorno do desenvolvimento intelectual) é um transtorno com início no período do desenvolvimento que inclui déficits funcionais, tanto intelectuais quanto adaptativos, nos domínios conceitual, social e prático. Os três critérios a seguir devem ser preenchidos DSM-5:

Déficits em funções intelectuais como raciocínio, solução de problemas, planejamento, pensamento abstrato, juízo, aprendizagem acadêmica e aprendizagem pela experiência confirmados tanto pela avaliação clínica quanto por testes de inteligência padronizados e individualizados.

Déficits em funções adaptativas que resultam em fracasso para atingir padrões de desenvolvimento e socioculturais em relação a independência pessoal e responsabilidade social. Sem apoio continuado, os déficits de adaptação limitam o funcionamento em uma ou mais atividades diárias, como comunicação, participação social e vida independente, e em múltiplos ambientes, como em casa, na escola, no local de trabalho e na comunidade. Início dos déficits intelectuais e adaptativos durante o período do desenvolvimento DSM-5 (2014, p. 77).

De acordo com o DSM-V (2014) a utilização de medidas através de QI não basta como sendo uma fonte suficiente para a medida precisa em que cada indivíduo se encontra, destacando que: “Escores de QI são aproximações do funcionamento conceitual, mas podem ser insuficientes para a avaliação do raciocínio em situações da vida real e do domínio de tarefas práticas" (p. 77).

Sendo classificado no manual como 317 (F70) Leve; 318.o (F 71 ) Moderada; 318.I ( $\left.F_{72}\right)$ Grave; 318.2 ( $\left.F_{73}\right)$ Profunda, siglas utilizadas pela categoria médica nas especificações em que cada indivíduo possa se classificar. Os vários níveis de gravidade segundo o DSM5 devem ser definidos com base no funcionamento adaptativos, e não em escores de QI, "uma vez que é o funcionamento adaptativo que determina o nível de apoio necessário. Além disso, medidas de QI são menos válidas na extremidade mais inferior da variação desse coeficiente" (p. 77).

No entanto o funcionamento adaptativo passa a ser o diferencial no que se refere ao desenvolvimento integral dos sujeitos com DI, os descritos abaixo demostram quais domínios e níveis de gravidade de acordo com o DSM-5 (2014, p. 78,79 e 8o). Os níveis de gravidade para DI (transtorno do desenvolvimento intelectual) podem ser descrita como:

Leve: Em crianças pré-escolares, pode não haver diferenças conceituais óbvias. Para crianças em idade escolar e adultos, existem dificuldades em aprender habilidades acadêmicas que envolvam leitura, escrita, matemática. Comparado aos indivíduos na mesma faixa etária com desenvolvimento típico, o indivíduo 


\begin{abstract}
mostra-se imaturo nas relações sociais. Por exemplo, pode haver dificuldade em perceber, com precisão, pistas sociais dos pares. Moderada: Durante todo o desenvolvimento, as habilidades conceituais individuais ficam bastante atrás das dos companheiros. Nos pré-escolares, a linguagem e as habilidades préacadêmicas desenvolvem-se lentamente. $O$ indivíduo mostra diferenças marcadas em relação aos pares no comportamento social e na comunicação durante o desenvolvimento. A linguagem falada costuma ser um recurso primário para a comunicação social $O$ indivíduo é capaz de dar conta das necessidades pessoais envolvendo alimentar-se, vestir-se, eliminações e higiene
\end{abstract}

Grave: Alcance limitado de habilidades conceituais. Geralmente, o indivíduo tem pouca compreensão da linguagem escrita ou de conceitos que envolvam números, quantidade, tempo e dinheiro. A linguagem falada é bastante limitada em termos de vocabulário e gramática. A fala pode ser composta de palavras ou expressões isoladas, com possível suplementação por meios alternativos. $\mathrm{O}$ indivíduo necessita de apoio para todas as atividades cotidianas, inclusive refeições, vestir-se, banhar-se e eliminação (2014, p. 78,79 e 80).

As mencionadas definições trazem em seus descritos os referidos défices existentes nas pessoas com DI nos respectivos níveis em que cada uma se encontra, dessa forma favorecendo no entendimento de que algumas áreas serão comprometidas, e de que formas poderá ser trabalhado a deficiência trazida por alguns sujeitos. Proporcionando aos profissionais um melhor entendimento para que assim seja possível a realização de intervenções nas relativas áreas que necessitam de maiores estímulos.

Dessa forma o conhecimento do que fazer, proporciona avanços no processo de inclusão e desenvolvimento integral das pessoas que forem acometidas pela DI, e ao mesmo tempo tiverem a oportunidade de serem agraciadas por profissionais juntamente com meio escolar para poder sanar em sua grande parte os défices acometidos pela deficiência.

\title{
CAUSAS DA DEFICIÊNCIA INTELECTUAL
}

As causas do porquê ou como se adquiriu uma DI percorreu por longas décadas sem uma explicação única, visto que devido à escassez de estudos e pesquisas detalhadas sobre a deficiência levaram algumas especulações a serem publicadas sem muita credibilidade e veracidade de causa, nos dias atuais sabe-se que existe algumas causas e não apenas uma em específico que pode fazer com que alguém seja considerado DI, dentre elas podemos citar: dano genético, dano causado dentro do útero, dano ocorrido no nascimento ou logo depois, acidentes e doenças e causas sociais entre outras, todas essas podendo levar os indivíduos a ter que aprender a viver com alguns défices existentes devido a prevalência da deficiência. 
A DI pode mostrar-se durante os primeiros anos de vida das crianças, devido a impossibilidade da aplicação de teste de QI já que esse teste não pode ser visto como uma variável única, podendo ser entendido como uma parte fundamental no processo do diagnóstico da deficiência, à idade dos mesmos não e suficiente para que seja possível a aplicação desse teste, ficando assim impossibilitado de se ter um diagnóstico mais preciso antes dos cinco anos de idade, acaba que se utilizando para as crianças que não conseguiram atingir os padrões referentes ao aprendizado esperados para a faixa etária, a denominação de atraso do desenvolvimento, podendo ser acarretados por problemas no aspecto da adaptação como no aprendizados dos sujeitos.

Algumas manifestações referentes ao diagnóstico da DI mais comum e com relação a possíveis atrasos no desenvolvimento da linguagem. Assim, passa a ser essencial que os profissionais da atenção básica que irão trabalhar com essas crianças, fiquem atentas a essas características, como também outros aspectos relacionados a déficit auditivo, atraso na fala, problemas na interação social dessas crianças, podendo ser melhor investigados se realmente tem a ver com problemas relacionados a DI Brasília (2018).

De acordo com o manual de Genética médica para não especialistas: o reconhecimento de sinais e sintomas, elaborado pelo Conselho Federal de Medicina, Brasília (2018, p. 17).

A prevalência global de DI varia de $1 \%$ a $3 \%$, ficando em torno de $1 \%$ nos países com renda alta, e de 2 a $3 \%$ nos de renda baixa e média, onde se concentra a maior parte da população infantil mundial e onde fatores deletérios do ambiente devem contribuir para aumentar o risco de ocorrência. Um total de $85 \%$ dos casos corresponde a DI leve, I0\% a DI moderada, $4 \%$ a DI grave e $2 \%$ a DI profunda. $\mathrm{Na}$ maioria dos registros há um predomínio do sexo masculino, com razão de sexo de I, 5:I. No Brasil, conforme dados do censo demográfico de 2010, há pelo menos 2,6 milhões ( $1,4 \%$ ) de pessoas com DI, sendo estimado que 800 mil têm DI de causa genética.

O manual apresenta algumas das características que prevalece com relação aos números descritos sobre a DI, mostrando características sobre a maior incidência que em países com baixo índice de desenvolvimento a incidência de pessoas acometidas pela DI é bem maior quando comparado a países com uma renda elevada. Podendo também deixar mencionado dados relativos sobre onde se concentra a maior quantidade de pessoas com DI que é em grau leve, como destacando que a maior incidência de pessoas com DI é por causas genéticas isso quando falamos de Brasil.

A etiologia sobre o processo de investigação da DI requer uma atenção e cuidados no processo de reconhecimento, dessa forma "essa avaliação inicial em geral é feita na 
atenção básica, por pediatras, clínicos gerais e médicos das equipes de saúde da família. Nessa etapa, a anamnese e o exame físico serão os habitualmente realizados pelo médico, considerando sempre a avaliação global do indivíduo” Brasília (2018, p. 28). Como também se buscar a realização de exames dismorfológico, que consiste na realização de uma avaliação mais criteriosa no paciente, buscando por sinais que identifique alguma síndrome genética.

A conjectura de que exista DI, surge em geral através da comprovação de alguns aspectos como: "atraso global do desenvolvimento neuropsicomotor; hipotonia; atraso de fala; déficit cognitivo; distúrbios de comportamento" (p. 28). Esses sinais existentes nas crianças surge como sinal de alerta para que se possa buscar junto aos profissionais, meios para se trabalhar os défices existentes em cada área específica. Sendo o diagnóstico mais comum nos primeiros anos, em idade escolar, fator melhor observável quando estes começam a apresentarem problemas geralmente relacionados a dificuldades de aprendizagem Brasília (2018).

As manifestações mais comuns, conforme a faixa etária, encontram-se na tabela abaixo:

Tabela $N^{\circ}$ 2: Manifestações mais comuns de atraso do desenvolvimento/DI conforme a faixa etária

\begin{tabular}{|c|c|}
\hline Faixa Etária & Manifestação \\
\hline Recém-nascido (o a 28 dias) & $\begin{array}{c}\text { Síndromes dismórficas, anomalias congênitas do } \\
\text { SNC e/ou disfunção orgânica afetando funções } \\
\text { vitais como alimentação. }\end{array}$ \\
\hline Lactente (28 dias a II meses) & $\begin{array}{c}\text { Interação reduzida com a mãe e/ou ambiente; } \\
\text { suspeita de déficit auditivo e/ou visual; atraso do } \\
\text { desenvolvimento motor e alterações de } \\
\text { comportamento. }\end{array}$ \\
\hline Infante (I2 a 36 meses) & $\begin{array}{c}\text { Atraso/dificuldade de fala; dificuldade de } \\
\text { interação social e padrões incomuns de } \\
\text { comportamento. }\end{array}$ \\
\hline Pré-escolar (3 a 7 anos) & $\begin{array}{c}\text { Atraso ou distúrbio de linguagem; déficit na } \\
\text { coordenação motora fina (dificuldade para } \\
\text { recortar, colorir, desenhar etc.); alterações de } \\
\text { comportamento como dificuldade de interação } \\
\text { social e agitação psicomotora. }\end{array}$ \\
\hline Escolar (8 a II anos) & $\begin{array}{c}\text { Dificuldade escolar, déficit de atenção, agitação } \\
\text { psicomotora, ansiedade, distúrbios de humor. }\end{array}$ \\
\hline
\end{tabular}

Manual de Genética médica para não especialistas: o reconhecimento de sinais e sintomas, elaborado pelo Conselho Federal de Medicina, Brasília (2018).

As crianças as quais nascem com síndrome genéticas já carregam consigo a DI, vindo a surgir conforme as mesmas começam seu processo de desenvolvimento, tanto os 
aspectos cognitivos como na interação social, podendo assim ser melhor facilmente detectado a presença da deficiência. Dessa forma podendo logo em seguida a busca pela melhor forma de inserção ao ambiente em que elas possam obterem ajuda para assim se desenvolverem de forma intensa, e cada vez mais diminuir a presença das dificuldades desencadeadas pela DI.

Uma vez que a obtenção do êxito no processo do diagnóstico da DI está relacionado a fatores da observação detalhada dos sujeitos no seu processo de desenvolvimento, dessa forma podendo assim encaminhar aos serviços adequados para a buscar de tratamento, e aos poucos buscando a inclusão no meio social, inclusive no contexto educacional.

A DI é classifica em leve, moderada, severa e profunda, isso relacionado ao funcionamento intelectual de cada indivíduo, segundo American Psychiatry Association APA (2014) o funcionamento adaptativo é o que é determinante com relação ao nível de apoio necessário para que esses sujeitos possam realizar diversas atividades. A DI foi percebida que sua condição seria melhorada com a percepção de suporte e não enxergando a mesma como deficiência estática, buscando a prestação de suporte, com isso foram deliberados em quatro níveis:

Apoio intermitente o apoio é oferecido conforme as necessidades do indivíduo. É caracterizado de natureza episódica, pois a pessoa nem sempre necessita de apoio. $\mathrm{O}$ apoio geralmente se faz necessário por períodos curtos durante transições ao longo da vida, como, por exemplo, perda do emprego ou uma crise médica aguda. $\mathrm{O}$ apoio intermitente pode ser de alta ou baixa intensidade.

Apoio limitado a intensidade de apoio é caracterizada por consistência ao longo do tempo. O tempo é limitado, mas não de natureza intermitente, podendo exigir poucos membros do staff e de custo menor, se comparado com outros níveis de apoio mais intensivos. São exemplos desse tipo de apoio o treinamento para o emprego no mercado competitivo por um tempo limitado ou o apoio na transição da vida escolar para a vida adulta. Apoio amplo o apoio amplo é caracterizado pelo apoio regular (por exemplo, apoio diário) em pelo menos alguns ambientes (por exemplo, no trabalho, na escola) e não por tempo limitado (por exemplo, apoio permanente nas atividades de vida diária). Apoio permanente, apoio caracterizado pela constância e alta intensidade. É oferecido nos ambientes onde a pessoa vive e é de natureza vital para sustentação da vida do indivíduo. $O$ apoio permanente tipicamente envolve mais membros do staff e é mais intensivo que o apoio por tempo limitado ou apoio amplo em ambientes específicos (ALMEIDA, 2012, p. 58).

Se fazendo necessário que não haja instrumentos padronizados para avaliarem os comportamentos adaptativos dos indivíduos, e os seus níveis de apoio necessário no processo de desenvolvimento. Visto que o teste de QI não é suficiente para indicar limitações relacionadas ao comportamento, se fazendo necessário outros suportes, já que o 
indivíduo está inserido em ambientes diversos, sendo todos necessário a adequação para o processo de inserção e adaptação no processo de inclusão.

\section{CONSIDERAÇÕES FINAIS}

A pessoa com deficiência sempre foi vista em todos as partes do mundo como sujeitos incapacitantes, pessoas que não eram providas de adquirir conhecimento nem tão pouco ter independência, essa percepção era tida por grande parcela da população em diversas partes do mundo. Aos poucos esses conceitos foram sendo desmistificados através da ciência e das inúmeras lutas sociais e através das políticas públicas inerentes a todos aqueles com DI.

A terminologia usada para descrever a pessoas com DI também sofreu mudanças e assim foi possível perceber e entender que esse sujeito não tem nenhuma doença que impeça o mesmo de ser independente, sua característica está associada a algumas limitações que podem ser superadas com estímulos e um bom processo educacional.

O espaço escolar vem sendo um grande diferencial na vida de todos aqueles que venham ser acometidos por alguma deficiência, no caso das crianças com DI esse tipo de espaço vem possibilitando mudanças essências tanto nos aspectos psicológicos, maturacional e na sua independência algo que a algumas décadas atrás era impossível imaginar alguém diagnosticado com DI poder estudar, trabalhar e realizar atividades que eram descritas apenas para pessoas sem nenhuma deficiência.

A mudança vem ocorrendo tanto nos aspectos da visibilidade externa como também percebidos e aceito pela família, colocando essas pessoas como seres capacitados necessitando apenas de estímulos e oportunidades para se desenvolverem e alcançarem tudo aquilo que foi colocado ao longo de anos como sendo algo inalcançável, como a participação das pessoas com deficiência dentro de todos os espaços e ambientes sociais que estes venham desejar pertencer e participar.

Portanto dentro da história da pessoa com DI é notório os avanços em diversos meios, porém muitas coisas precisam ainda serem organizadas para que todos aqueles que tenham a referida deficiência possam usufruir de forma igualitária de tudo aquilo que desejem, sem serem rotulados nem tão pouco impedidos de realizar. Dessa forma se faz necessário que as políticas públicas venham cada vez mais serem reformuladas para assim 
alcançar e favorecer a todos aqueles que necessitem de forma condizente a sua especificidade.

\section{REFERÊNCIAS}

AAIDD. Americanassociation on intellectual and developmental disabilities- Definition of Intellectual Disability. Washington, D.C: AAIDD (2016). Disponível em: https://www.aaidd.org/intellectual-disability/definition\#.Vi8LLvkrKUk. Acesso em o9 de janeiro de 2021.

Alles, E.P.; Castro. S.F.; Boueri. I. Z. Educação inclusiva e contexto social [recurso eletrônico]: questões contemporâneas 2 / Organizador Willian Douglas Guilherme PontaGrossa(PR):AtenaEditora(2019).Disponívelem:https://www.atenaeditora.com.br/w p-content/uploads/2019/o6/E-book-EducacaoInclusiva-e-Social.pdf. Acesso em is de janeiro de 2020 .

APA. American psychiatric association. Manual diagnóstico e estatístico de transtorno mentais: DSM-5. Porto Alegre: Artmed (2014).

Almeida, M. A. (Org.). Deficiência intelectual: realidade e ação. Secretaria da Educação. Núcleo de Apoio Pedagógico Especializado - CAPE. São Paulo: SE (2012).

Brasília. Genética médica para não especialistas: o reconhecimento de sinais e sintomas. Conselho Federal de Medicina. CFM(2018). Disponível em:https://apaepr.org.br/uplo.pdf. Acesso em: 03 de janeiro de 202I.

Brites, C. Deficiência intelectual entre mitos e verdade. Neuro saber Ltda ME (2019). Disponivel em: https://neurosaber.com.br intelectual-e-desenvolvimental-did/. Acesso em 03 de janeiro de 2021.

OMS - Organização Mundial de Saúde. Declaração de Montreal sobre Deficiência Intelectual. Montreal, Canadá, 4-6 outubro 2004.

OMS - Organização mundial da saúde Orientações referentes ao combate à disseminação e proteção para evitar o contágio da doença (covid-19) (2020). Disponível em: https://www.paho.org/bra/index.php?option=com_content\&view=article\&id=6138:covidI9-oms-atualiza-guia-com-recomendacoes-sobre-uso-de-mascaras $\&$ Itemid=812. Acesso em: 04 de outubro de 2021. 